Bio - grafía. Escritos sobre la Biología y su Enseñanza. ISSN 2027-1034

Edición Extraordinaria. p.p. 848 - 856

Memorias del IX Encuentro Nacional de Experiencias en Enseñanza de la Biología y la Educación Ambiental. IV Congreso Nacional de Investigación en Enseñanza de la Biología.

\title{
LAEVOLUCIÓN BIOLÓGICA Y LOS ELEMENTOS ESTRUCTURALES DE SU MODELO TEÓRICO
}

\section{BIOLOGICAL EVOLUTION AND THE STRUCTURAL ELEMENTS OF HIS THEORETICAL MODEL}

\author{
Martínez Hernández, María de la Luz ${ }^{1}$ \\ Rodríguez-Pineda, Diana Patricia ${ }^{2}$
}

\section{RESUMEN.}

Diversas investigaciones en el campo de la Educación en Ciencias reportan que los perfiles de los docentes de Biología de secundaria son diversos: biólogos, químicos, médicos, odontólogos, entre otros, en su mayoría no tienen formación inicial como profesores, para la enseñanza de esta asignatura, esto es importante pues se requiere de un especialista que vea los aspectos fundamentales de los procesos vitales, característicos de los seres vivos y la diversidad como resultado de la evolución. Dado que algunos investigadores mencionan que la enseñanza de la evolución resulta compleja, se considera fundamental conocer la comprensión que tienen los docentes respecto al tema, lo que resulta relevante conocer: ¿Cuál es el pensamiento del profesorado sobre el Modelo Teórico de Evolución Biológica -MTEB-?, por tanto, el propósito es construir una herramienta categorial y analítica que se utilice para identificar el MTEB del docente. Se inició con una revisión bibliográfica especializada en Biología evolutiva para relacionar los componentes comunes de las teorías de Lamarck, Darwin y Sintética, posteriormente a partir del concepto de modelo teórico se identificaron los elementos estructurales del MTEB -entidades y sus propiedades, relaciones entre entidades y condiciones- $\mathrm{y}$ por último se integró una tabla con cada uno de los componentes y su descripción de la familia del MTEB. Finalmente se obtuvo como producto una herramienta categorial y analítica que permitirá discriminar al interior del MTEB, los elementos estructurales con detalle con los cuales se podrá construir y analizar un instrumento que se aplicará a los profesores de secundaria.

PALABRAS CLAVE: Modelos, modelos teóricos, evolución biológica, profesores de biología, secundaria.

\footnotetext{
1 SEP-CSES/UPN Doctorado en Educación, Línea Educación en Ciencias. fluzma@hotmail.com

${ }^{2}$ Universidad Pedagógica Nacional. dpineda@upn.mx
} 
Bio - grafía. Escritos sobre la Biología y su Enseñanza. ISSN 2027-1034

\section{Edición Extraordinaria. p.p. 848 - 856}

Memorias del IX Encuentro Nacional de Experiencias en Enseñanza de la Biología y la
Educación Ambiental. IV Congreso Nacional de Investigación en Enseñanza de la
Biología.

ABSTRACT.

Many research in the field of Science Education report that the profiles of the high school biology teachers are diverse: biologists, chemists, doctors, dentists, among others, most of them do not have initial training as teachers, for the teaching of this subject, this is important because it requires a specialist to see the fundamental aspects of the vital processes, characteristic of living beings and the diversity because of evolution. Given that some researchers mention that the teaching of evolution is complex, it is essential to know the understanding of teachers on the subject, which is relevant to know, what is the thought of the faculty on the Theoretical Model of Biological Evolution -MTEB-? therefore, the purpose is to build a categorial and analytical tool that is used to identify the teacher MTEB. It began with a review of the literature specialized in evolutionary biology to relate the common components of the theories of Lamarck, Darwin and synthetic, subsequently based on the concept of theoretical model identified the structural elements of the MTEB entities and their properties, relationships between entities and conditions- and finally joined a table with each of the components and their description of the family of the MTEB. The product was a categorial and analytical tool that will allow to discriminate against the inside of the MTEB, structural elements in detail with which you will be able to construct and analyze an instrument to be applied to the secondary school teachers.

KEYWORDS: models, theoretical models, biological evolution, biology teachers, secondary

\section{INTRODUCCIÓN}

A lo largo de la vida de la escuela secundaria en México, como lo han reportado Martínez y Rodríguez-Pineda (2015) la formación de los docentes que imparten biología sigue la tendencia de mayor frecuencia los que no tienen formación inicial como profesor universitario- y menor a los que tienen formación inicial como profesor -normalista- esto no es exclusivo de este país sino también de algunos otros de Europa y Latinoamérica. Los perfiles de los docentes son diversos existen biólogos, químicos, licenciados en ciencias naturales, médicos, odontólogos, tal diversidad parece tener consecuencias pedagógicas, así como el bajo nivel académico de los alumnos. Rico (2006) menciona que sólo el $27 \%$ de quienes enseñan tengan como disciplina a la Biología, esto es preocupante pues se requiere de un especialista que vea los aspectos fundamentales de los procesos vitales, característicos de los seres vivos y la diversidad como resultado de la evolución biológica.

Un tema que está en la asignatura de Biología es la diversidad de los seres vivos, el cual se constituye en el eje fundamental para entenderla como ciencia articulada a partir de la evolución biológica. La enseñanza de la evolución resulta compleja, se ha discutido en la literatura la pertinencia de abordar este tema en la educación básica (Jiménez-Aleixandre, 1989; Salgado, 2013), además algunos estudios realizados con estudiantes de diferentes 
Bio - grafía. Escritos sobre la Biología y su Enseñanza. ISSN 2027-1034

\section{Edición Extraordinaria. p.p. 848 - 856}

Memorias del IX Encuentro Nacional de Experiencias en Enseñanza de la Biología y la Educación Ambiental. IV Congreso Nacional de Investigación en Enseñanza de la Biología.

niveles educativos relacionados con este tema (Jiménez-Aleixandre, 1989; Galli, 2011) reportan que sus explicaciones sobre la evolución desde un enfoque histórico, no corresponden con los postulados de la teoría moderna -incluida la sintética-de donde se desprende que la mayor parte de los alumnos en su paso por el sistema educativo no han alcanzado este propósito que, para la enseñanza de Biología es fundamental.

Las caracterizaciones más comunes referidas a las explicaciones del docente que enseña ciencias sobre la evolución biológica se han elaborado desde la perspectiva de las 'concepciones alternativas', todas ellas coinciden en que las expresiones que dan los docentes son: teológicas -desde sus génesis- y teleológicas -desde su finalidad-, con frecuencia alojadas en el pensamiento similar al de Lamarck (Jiménez-Aleixandre, 1992; Sánchez, 2000; Rico, 2006),

En este sentido, recuperando lo planteado por Bahamonde (2007), que los profesores tengan concepciones alternativas entorno al proceso de la teoría de la evolución, está relacionado con su comprensión respecto al tema, dicha comprensión es producto de los modelos teóricos que han incorporado los profesores en la construcción de sus modelos explicativos, éstos pueden estar influenciados por su perfil de formación profesional (Martínez y Rodríguez-Pineda, 2015).

Dicho lo anterior se considera fundamental conocer la comprensión que tienen los docentes respecto al tema, por lo que resulta relevante identificar: ¿Cuál es el pensamiento del profesorado sobre el Modelo Teórico de Evolución Biológica MTEB-? Por tanto, el propósito de este trabajo es: Construir una herramienta categorial y analítica, a partir de los elementos estructurales del MTEB -entidades y sus propiedades, relaciones entre entidades $y$ condiciones- que se utilice para identificar el MTEB del docente.

\section{REFERENTES TEÓRICOS}

Una forma emergente de acercarse por inferencia al pensamiento de los teóricos y de sus productos son los modelos, lo que es importante conocer la articulación de la estructura de una teoría y su validación como propone Giere (1992), así mismo señala a la teoría como una serie de enunciados referidos a explicar la realidad, ésta tiene limitaciones y que esas limitaciones deben superarse. Un modo de hacerlo es emplear modelos para explicarse la realidad, luego un modelo es una manera de representar la realidad de forma más completa y dinámica, más que una serie de enunciados ya que un enunciado se limita al uso del lenguaje oral o escrito en tanto el modelo es una abstracción que tiene infinidad de posibilidad para describir, explicar y predecir los fenómenos de la realidad.

Un modelo puede ser concretado de forma física, como una maqueta o un maniquí anatómico (Adúriz-Bravo, 2011; Gómez, 2008). Sin embargo, esas concreciones pasan primero por ser pensadas, son abstracciones que construyen y representan un fenómeno, lo validan según criterios epistemológicos de verdad y lo operan, todo en la mente de las personas, esto lo pueden concretar ya sea en un escrito, en una expresión de lenguaje o 
Bio - grafía. Escritos sobre la Biología y su Enseñanza. ISSN 2027-1034

\section{Edición Extraordinaria. p.p. 848 - 856}

Memorias del IX Encuentro Nacional de Experiencias en Enseñanza de la Biología y la Educación Ambiental. IV Congreso Nacional de Investigación en Enseñanza de la Biología.

bien de forma física. Al primero se le denomina modelo teórico también denominado modelo científico o erudito.

Como todo modelo teórico es importante identificar en él, las entidades, relaciones y condiciones o reglas de operación del mismo -de acuerdo con Lesh y Doerr (2003) citados por Schwarz, et al. (2009). Un modelo ontogenéticamente evoluciona en el sujeto que lo construye y porta, pero históricamente la forma en que se representa y concibe a un fenómeno, objeto de estudio, también evoluciona y forma un linaje de modelos -una familia de modelos- (Tamayo y Sanmartí, 2007), al respecto González-Galli (2010, p.227) menciona:

“...la biología evolutiva pretende dar cuenta de un amplio conjunto de fenómenos, para la cual recurre a un diverso arsenal de modelos teóricos. Desde la perspectiva epistemológica, podemos pensar que una teoría es un conjunto de modelos, (Giere, 1992), así podemos ver a la teoría evolutiva como una familia de modelos".

La evolución biológica se considera una teoría integrativa puesto que intenta unificar el saber biológico en la explicación de la evolución, es decir, a partir de los descubrimientos de las disciplinas biológicas -como genética, biología molecular, paleontología, biología del desarrollo, sistemática, etc.- explica tales fenómenos como: la diversidad, la adaptación, la extinción, el origen de novedades morfológicas y fisiológicas, la especiación; sus propósitos centrales son unir la historia de la evolución y esclarecer sus mecanismos.

En el estudio de la historia de la evolución se hacen inferencias sobre acontecimientos del pasado a partir del registro fósil (paleontología) y de las relaciones filogenéticas, que a su vez se deducen del estudio de los patrones existentes (sistemática y genética molecular). Los mecanismos que se consideran fundamentales en el proceso evolutivo son: mutación, selección, deriva genética, migración y recombinación, éstos se combinan con variable importancia en los diferentes niveles y unidades de evolución (Ruiz y Ayala, 2002).

\section{METODOLOGÍA}

Para la construcción de la herramienta categorial y analítica, se inició con una revisión bibliográfica especializada en Biología evolutiva (Ruiz y Ayala, 2002; Ruiz, 2013), particularmente los planteamientos de las teorías de Lamarck, Darwin y Sintética para relacionar los componentes comunes, posteriormente a partir del concepto de modelo teórico se identificaron los elementos estructurales del MTEB -entidades y sus propiedades, relaciones entre entidades y condiciones - y por último se integró una tabla con cada uno de los componentes -categorías de análisis- y su descripción de la familia del MTEB.

Esta herramienta será la que posteriormente se utilizará tanto para construir y analizar el instrumento que se aplicará y, que permitirá identificar el MTEB del profesor especialista o no en el tema (Ruiz y Ayala, 2002). El sistema categorial y analítico fue discutido por 
Bio - grafía. Escritos sobre la Biología y su Enseñanza. ISSN 2027-1034

Edición Extraordinaria. p.p. 848 - 856

Memorias del IX Encuentro Nacional de Experiencias en Enseñanza de la Biología y la Educación Ambiental. IV Congreso Nacional de Investigación en Enseñanza de la Biología.

investigadoras especializadas en el estudio para después ser validado mediante jueceo de expertos.

\section{RESULTADOS}

Se identificaron los elementos estructurales en los que se tiene como entidad a los seres vivos -unidad biológica evolutiva-, emergen tres propiedades de la entidad -variabilidad, adaptabilidad y heredabilidad-, se obtienen cuatro relaciones entre entidades competencia, parentesco, descendencia y especiación- y, por último, las dos condiciones para que el modelo opere -gradualismo y mecanismos de la evolución-, en total son diez componentes que forman parte de la familia del MTEB (ver Figura 1).

Figura 1. Elementos estructurales del MTEB

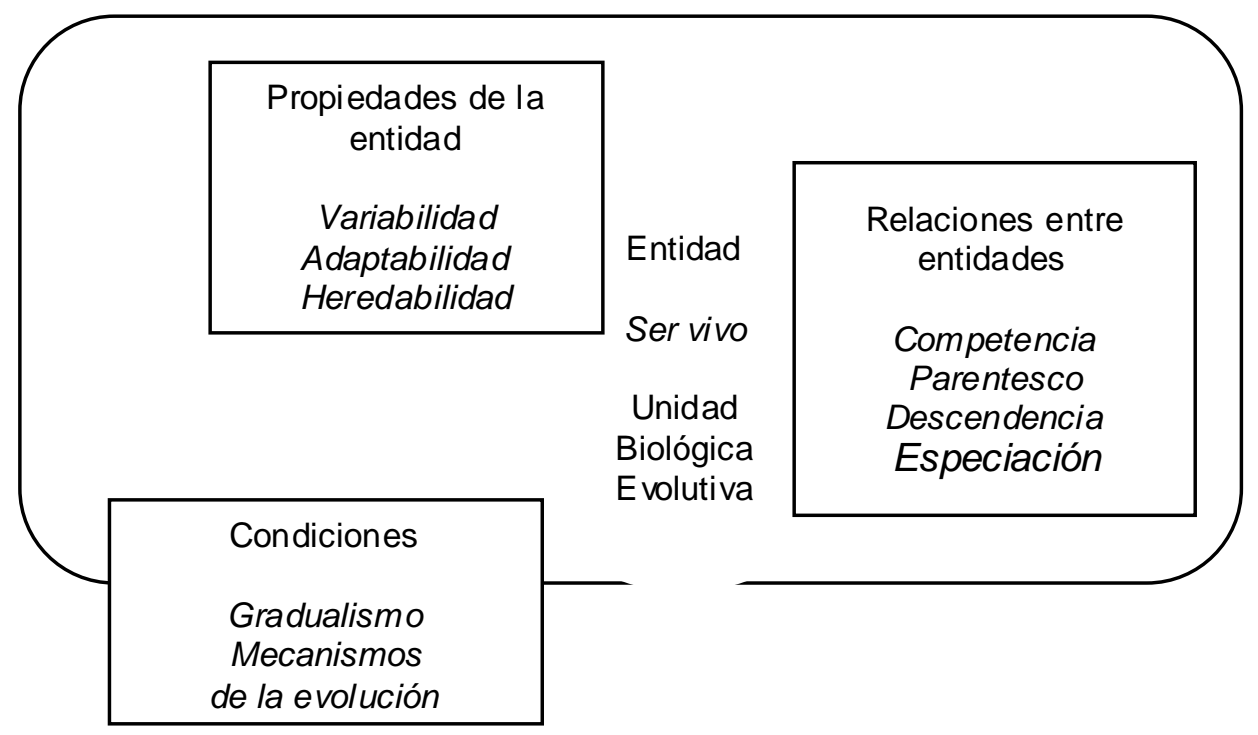

Con esta estructura se asume que se pueden inferir otros modelos teóricos, sin que la similitud con algún modelo preexistente sea un criterio de validez, sino que permitirá ubicarlo según sus características propias, valorando su particularidad.

Posteriormente se integró una tabla de los elementos estructurales con cada uno de sus componentes de la familia del MTEB y su descripción (ver Tabla 1). 
Bio - grafía. Escritos sobre la Biología y su Enseñanza. ISSN 2027-1034

\section{Edición Extraordinaria. p.p. 848 - 856}

Memorias del IX Encuentro Nacional de Experiencias en Enseñanza de la Biología y la Educación Ambiental. IV Congreso Nacional de Investigación en Enseñanza de la Biología.

Tabla 1. Elementos y componentes estructurales detallado del MTEB

\begin{tabular}{|c|c|c|}
\hline $\begin{array}{c}\text { Elementos } \\
\text { del } \\
\text { Modelo } \\
\text { Teórico }\end{array}$ & $\left|\begin{array}{c}\text { Componentes } \\
\text {-Categorías- }\end{array}\right|$ & Descripción \\
\hline Entidad & $\begin{array}{l}\text { Ser vivo } \\
\text { Unidad } \\
\text { biológica } \\
\text { evolutiva }\end{array}$ & $\begin{array}{l}\text { Sistema que intercambia materia y energía con el medio y como } \\
\text { resultado de ello modifica el medio, capta estímulos del medio y } \\
\text { responde a ellos, proviene de otros seres vivos y puede reproducirse } \\
\text { y transferir su características a sus descendientes y está constituido } \\
\text { por una o muchas unidades estructurales que llamamos células, cada } \\
\text { una de las cuales tiene a su vez las mismas propiedades del todo, } \\
\text { además evolucionan al incrementarse sus unidades de organización } \\
\text { y niveles de complejidad (García, 2005). }\end{array}$ \\
\hline \multirow{3}{*}{$\begin{array}{c}\text { Propiedades } \\
\text { de la } \\
\text { Entidad }\end{array}$} & Variabilidad & $\begin{array}{l}\text { Capacidad que tienen los seres vivos de presentar alternativas de un } \\
\text { mismo carácter }\end{array}$ \\
\hline & Adaptabilidad & $\begin{array}{l}\text { Capacidad que tienen los seres vivos para responder a las presiones } \\
\text { del medio }\end{array}$ \\
\hline & Heredabilidad & Capacidad que tienen los seres vivos para transmitir sus caracteres \\
\hline \multirow{4}{*}{$\begin{array}{c}\text { Relaciones } \\
\text { entre } \\
\text { entidades }\end{array}$} & Competencia & Interacción entre los seres vivos por los mismos recursos \\
\hline & Parentesco & $\begin{array}{l}\text { Lazos o vínculos de linaje que se tienen entre los seres vivos o } \\
\text { unidades evolutivas }\end{array}$ \\
\hline & Descendencia & Producto de la reproducción de las generaciones progenitoras \\
\hline & Especiación & Génesis de nuevas especies (a partir de las ya existentes) \\
\hline \multirow{2}{*}{ Condiciones } & Gradualismo & $\begin{array}{l}\text { Tiempo y complejidad Procesos evolutivos que se dan en forma } \\
\text { continua a través del tiempo, ir de lo sencillo a lo complejo en forma } \\
\text { continua }\end{array}$ \\
\hline & $\begin{array}{c}\text { Mecanismos } \\
\text { de la } \\
\text { evolución }\end{array}$ & $\begin{array}{l}\text { Diversidad de procesos que se encuentran a través de la evolución } \\
\text { biológica }\end{array}$ \\
\hline
\end{tabular}


Bio - grafía. Escritos sobre la Biología y su Enseñanza. ISSN 2027-1034

\section{Edición Extraordinaria. p.p. 848 - 856}

Memorias del IX Encuentro Nacional de Experiencias en Enseñanza de la Biología y la Educación Ambiental. IV Congreso Nacional de Investigación en Enseñanza de la Biología.

\section{DISCUSIÓN}

Los trabajos previos sobre acceso a la información de los docentes que enseñan biología en educación secundaria, para conocer sobre sus conocimientos, sus ideas alternativas 0 su estructura mental han empleado cuestionarios cerrados, de selección múltiple, de situaciones o construcción de mapas mentales a partir de cuestionarios. En todos los casos se toma como base la similitud que logre el sujeto de estudio con una teoría maestra o erudita. Paz (1999), González-Galli (2011) aducen que lo interesante no es ver similitudes entre un modelo erudito y su reflejo casi especular como sinónimo de conocimiento de una teoría, sino que la construcción conceptual que logre el sujeto de estudio es válida por sí misma y tiene características propias que han de evolucionar al seguir ahondando en su conocimiento (Tamayo y Sanmartí; 2007, Gómez, 2008).

Una forma de percibir el por qué se obtienen siempre resultados similares a la teoría transformista de Lamarck preferentemente, aduce que el docente tiene 'obstáculos epistemológicos' (González-Galli, Adúriz-Bravo y Meinardi, 2005) o 'conocimientos socialmente estabilizados' (Jiménez-Aleixandre, 1992) que hay que superar. Incidir en el pensamiento del profesor de biología de educación secundaria sobre el MTEB como rector de la acción docente no ha sido investigado de forma abundante (Martínez y Rodríguez-Pineda, 2016).

Para conocer el pensamiento modélico del profesor sobre la evolución biológica es necesaria una herramienta analítica que describa y valore éstos no que los identifique y clasifique, que reconozca su diversidad no que la señale ni etiquete. En este sentido es donde se busca proponer el uso de matrices con componentes estructurales que permitan valorar la diversidad del pensamiento docente desde un enfoque de modelos, particularmente en este caso ejemplificado con la familia de modelos de la evolución biológica.

\section{CONCLUSIONES}

Se construyó una herramienta analítica detallada y completa que permite discriminar al interior de los MTEB los elementos estructurales, con los cuales se podrá construir y analizar un instrumento que se aplicará a los profesores que permita identificar el MTEB del profesor de secundaria. Se propone el uso de matrices con componentes estructurales que permitan valorar la diversidad del pensamiento docente desde un enfoque de modelos. Con ello se afirma que toda teoría es susceptible de disgregarse para su análisis en sus componentes estructurales para reconocer: entidades y sus propiedades, relaciones entre entidades y condiciones.

Una forma emergente de acercarse por inferencia al pensamiento de los teóricos y de sus productos son los modelos, conocer la articulación de la estructura de una teoría y su validación como propone Dulsch (1997) ha sido empleada, pero el uso de modelos como propone Giere (1992) no ha sido explotado, y ese es el aporte de este trabajo, proponer 
Bio - grafía. Escritos sobre la Biología y su Enseñanza. ISSN 2027-1034

\section{Edición Extraordinaria. p.p. 848 - 856}

Memorias del IX Encuentro Nacional de Experiencias en Enseñanza de la Biología y la Educación Ambiental. IV Congreso Nacional de Investigación en Enseñanza de la Biología.

una herramienta operativa de corte analítica que permita inferir los modelos de los profesores a partir de aspectos concretos.

\section{AGRADECIMIENTOS}

Este trabajo fue posible gracias a una beca-comisión para posgrado, otorgada por la Coordinación Sectorial de Educación Secundaria de la SEP.

\section{BIBLIOGRAFÍA}

Adúriz-Bravo, A. (2011). Concepto de modelo científico: una mirada epistemológica de su evolución. En L. Galagovsky (coord.) Didáctica de las ciencias naturales. El caso de los modelos científicos (141-161). Buenos Aires: Logar.

Bahamonde, N. (2007). Los modelos de conocimiento científico escolar de un grupo de maestras de educación infantil: un punto de partida para la construcción de "islotes interdisciplinarios de racionalidad" y "razonabilidad" sobre la alimentación humana. (Tesis Doctoral). Barcelona: Universitat Autònoma de Barcelona.

García, M.P. (2005). Los modelos como organizadores del currículo de Biología. Enseñanza de las ciencias. Número Extra. VII Congreso.

Giere, R. (1992). La explicación de la ciencia: Un acercamiento cognoscitivo. México: Consejo Nacional de Ciencia y Tecnología.

Gómez, A. (2008). Construcción de explicaciones multimodales: ¿qué aportan los diversos registros semióticos? Revista Latinoamericana de Estudios Educativos, 4(2), 83-99.

González-Galli, L. (2010). La teoría de la evolución. En Meinardi. E.; González-Galli, L.; Revel, A. y Plaza M.V. Educar en Ciencias (225-259). Argentina: Paidós,

González-Galli, L. (2011). Obstáculos para el aprendizaje del modelo de evolución por selección natural. (Tesis Doctoral). Argentina: Universidad de Buenos Aires

González-Galli, L; Adúriz-Bravo, A y Meinardi, Elsa. (2005). El modelo cognitivo de ciencia y los obstáculos en el aprendizaje de la evolución biológica. Enseñanza de las Ciencias, Número Extra, VII. Congreso.

Jiménez-Aleixandre, M. P. (1992). Thinking about theories or thinking with theories? a classroom study with natural selection. International Journal of Science Education, 14(1), 51-61.

Martínez, L. y Rodríguez-Pineda, D.P. (2015). La formación profesional de los profesores de secundaria que enseñan Biología en México: el caso del Distrito Federal. Bio-grafía. 
Bio - grafía. Escritos sobre la Biología y su Enseñanza. ISSN 2027-1034

Edición Extraordinaria. p.p. 848 - 856

Memorias del IX Encuentro Nacional de Experiencias en Enseñanza de la Biología y la Educación Ambiental. IV Congreso Nacional de Investigación en Enseñanza de la Biología.

Escritos sobre la Biología y su enseñanza. Número extraordinario, III Congreso, 970981. DOI: http://dx.doi.org/10.17227/20271034.vol.0num.0bio-grafia970.981

Martínez, L. y Rodríguez-Pineda, D.P. (2016). La evolución biológica y el pensamiento del profesor de secundaria. Tecné y Episteme y Didaxis: TED, Número Extraordinario, VII. Congreso, 473-479.

Rico, C. (2006). Las Concepciones Alternativas de los Profesores de Educación Secundaria sobre la Evolución en los Seres Vivos. (Tesis de Maestría). México: Facultad de Ciencias-UNAM.

Ruiz, R. y Ayala, F. (2002). De Darwin al DNA y el origen de la humanidad: la evolución y sus polémicas. México: Fondo de Cultura Económica.

Ruiz, R. (2013). Grandes Maestros. UNAM. Daruinismo. Su significado, su impacto. Recuperado de: http://www.grandesmaestros.unam.mx/curso-disponible/darwinismosu-significado-su-impacto/

Tamayo, O y Sanmartí, N. (2007). High-school student's conceptual evolution of the respiration of the respiration concept from the perspective of Giere's cognitive science model. International Journal of Science Education. 29(2), 215-248.

Schwarz, C., Reiser, B., Davis, E., Kenyon, L., Achér, A., Fortus, D., Shwartz, Y., Hug, B. y Krajcik, J. (2009). Developing a learning progression for scientific modeling: making scientific modeling accessible and meaningful for learners. Journal of Research 